\title{
Nanotechnology-based drug delivery systems in orthopedics
}

\author{
Eylem Güven, $\mathrm{PhD}$ (1)
}

Department of Nanotechnology and Nanomedicine, Hacettepe University, Ankara, Turkey

Nanotechnology is a multidisciplinary branch of science which involves the manipulation and control of matter at the nanometer scale to produce new structures, materials, and devices. The concept of nanotechnology was first introduced by Richard P. Feynman ${ }^{[1]}$ in 1959. Since then, nanotechnology has become a research area which promises advancements in many aspects of human life, including electronics, agriculture, transportation, food industry, communication, energy, biological sciences, and medicine. Nanomedicine, the application of nanotechnology in the field of medicine, has the potential to make a great impact on human health and has already impacted and reshaped many aspects of clinical practice and research. Nanotechnology-based materials with unique physical, chemical, and biological characteristics offer a variety of new approaches for clinical practices ranging from prevention to treatment and diagnosis of many diseases and conditions. ${ }^{[2-5]}$ Among all the applications of nanotechnology in medicine, nano-based drug

Received: December 04, 2020

Accepted: January 03, 2021

Published online: January 06, 2021

Correspondence: Eylem Güven, PhD. Hacettepe Üniversitesi, Nanoteknoloji ve Nanotıp Anabilim Dalı, 06800 Beytepe, Ankara, Türkiye.

E-mail: eylemoz@hacettepe.edu.tr

Doi: 10.5606/ehc. 2021.80360

Citation: Güven E. Nanotechnology-based drug delivery systems in orthopedics. Jt Dis Relat Surg 2021;32(1):267-273.

(2021 All right reserved by the Turkish Joint Diseases Foundation

This is an open access article under the terms of the Creative Commons Attribution-NonCommercial License, which permits use, distribution and reproduction in any medium, provided the original work is properly cited and is not used for commercial purposes (http://creativecommons.org/licenses/by-nc/4.0/).

\section{ABSTRACT}

In recent years, nanotechnology has led to significant scientific and technological advances in diverse fields, specifically within the field of medicine. Owing to the revolutionary implications in drug delivery, nanotechnology-based drug delivery systems have gained an increasing research interest in the current medical field. A variety of nanomaterials with unique physical, chemical and biological properties have been engineered to develop new drug delivery systems for the local, sustained and targeted delivery of drugs with improved therapeutic efficiency and less or no toxicity, representing a very promising approach for the effective management of diseases. The utility of nanotechnology, particularly in the field of orthopedics, is a topic of extensive research. Nanotechnology has a great potential to revolutionize treatment, diagnostics, and research in the field of orthopedics. Nanophase drug delivery has shown great promise in their ability to deliver drugs at nanoscale for a variety of orthopedic applications. In this review, we discuss recent advances in the field of nanostructured drug delivery systems for orthopedic applications.

Keywords: Drug delivery, nanomedicine, nanotechnology, orthopedics.

delivery systems have attracted a significant research interest thanks to its great translational value. ${ }^{[6-10]}$ Owing to the rapid developments in nanotechnology, research in the field of controlled drug delivery has experienced an extraordinary progress. Nano drug delivery systems provide a new drug delivery method for the treatment of various diseases and demonstrate numerous advantages over traditional drug delivery systems. The nanoscale manipulation provides site-specific targeting and delivery, as well as the controllable release of drugs, genes, and imaging agents. $^{[11,12]}$ A variety of nano-based drug delivery systems have been recently developed from different materials such as lipids, polymers, metals, inorganic materials, and small molecules. ${ }^{[13]}$ Among the broad spectrum of nano-based drug delivery applications, orthopedics is one of the most active areas. 
In the past few decades, the emergence of nanotechnology has affected and reshaped every aspect of orthopedic research and practice. Nanotechnology has an exceptional potential for orthopedic applications owing to its unique ability to create materials and devices with extraordinary physicochemical, mechanical and biological properties. ${ }^{[14]}$ It has been utilized in a number of novel approaches including tissue engineering for bone regeneration, targeted drug delivery, surface modification of prosthesis and implants, and diagnostics in orthopedics. ${ }^{[15-19]}$ In particular, nanotechnology-based drug delivery systems have influenced many areas of orthopedics. In the current review, we discuss recent advances on nano-based drug delivery systems in the field of orthopedics.

\section{NANO-BASED DRUG DELIVERY SYSTEMS AND THEIR USES IN ORTHOPEDICS}

Drug delivery systems are engineered structures used to protect, transport, and release a pharmaceutical compound in a controlled manner. Compared to traditional drugs, nanotechnology-based drug delivery systems have several advantages, including high surface area-to-volume ratio for efficient drug loading, improved targeting due to their small size allowing them to overcome biological barriers, enhanced drug solubility and physical stability in biological media, ease of surface functionalization to sense, image, diagnose and deliver pharmaceuticals by the conjugation of biological targeting moieties, controlled dissolution rates/drug bioavailability, reduced adverse side effects, and size similarity to interact with, and modulate biological component. ${ }^{[20-22]}$

Various nanotechnology-based drug delivery vehicles, including polymeric nanoparticles, lipid nanoparticles, dendrimers, quantum dots, carbon nanotubes, and metallic nanoparticles have brought great advancements in the field of drug delivery, as well as the entire medical field. ${ }^{[23-27]}$ These delivery strategies have been applied for the treatment of bone-related diseases for increasing treatment efficiency and specificity of conventional clinical therapies. Nanoparticles and nano-based scaffolds have been widely used for the treatment of bone-related diseases including osteoarthritis, osteosarcoma, cancer bone metastasis, osteoporosis, bone infections and inflammatory diseases, as well as for bone tissue repair and regeneration. ${ }^{[28-32]}$

\section{Osteoarthritis}

Osteoarthritis is one of the most common joint diseases and a leading cause of disability, with a growing rate in prevalence worldwide. Current treatment options for osteoarthritis are limited, and many of these primarily focus on pain relief rather than modifying the disease progression. The major limitations for systemically administered osteoarthritis drugs are rapid clearance after intra-articular injection, limited cartilage targeting, and severe side effects due to multiple injections of high-dose drugs. ${ }^{[33]}$ Nanotechnology-based drug delivery systems have been explored for improving the pharmacodynamics and pharmacokinetics of osteoarthritis drugs through targeted and sustained therapeutic action with fewer systemic adverse effects and longer-term benefits. ${ }^{[34-38]}$

Maudens et al. ${ }^{[39]}$ developed kartogenin nanocrystal-polymer particles for effective treatment of osteoarthritis with a very high drug loading over an extended period of time as a controlled drug release system. Kartogenin is a small heterocyclic molecule with the ability of cartilage protection and regeneration. ${ }^{[40]}$ Polymeric particles loaded with kartogenin nanocrystals demonstrated a higher bioactivity than kartogenin solution in a murine mechanistic osteoarthritis model, representing a novel and innovative extended drug delivery system. ${ }^{[39]}$ In a study by Kang et al., ${ }^{[41]}$ thermoresponsive nanospheres based on chitosan oligosaccharide conjugated pluronic F127 grafting carboxyl group were synthesized for the simultaneous and independent dual release of kartogenin and diclofenac in a single system for combined osteoarthritis therapy. The nanospheres demonstrated initial burst release of diclofenac and sustained release of kartogenin in response to temperature change independently. In vitro and in vivo experiments suggested that these thermoresponsive nanospheres provide a dual function of anti-inflammatory and chondroprotective effects in the treatment of osteoarthritis. ${ }^{[41]}$ A cationic multi-arm avidin nano-construct was also designed and reported by He et al. ${ }^{[42]}$ for intra-cartilage delivery of a broad array of small molecule osteoarthritis drugs and their combinations to chondrocytes. Its small size and optimal positive charge enable avidin to penetrate deep into the cartilage. ${ }^{[4]]}$ Avidin nanoconstruct as an intra-cartilage drug delivery system may have potential to deliver combination of drugs with only a single injection eliminating toxicity issues and maintain sustained drug release within the joint for efficient and safe osteoarthritis treatment. ${ }^{[42]}$

\section{Orthopedic oncology}

Osteosarcoma is the most common primary malignant bone neoplasm. Chemotherapy is an important treatment of osteosarcoma, but limited for 
severe side effects and drug resistance. ${ }^{[44]}$ Advances in nanotechnology have led to the development of multifunctional nanomaterials as diagnostic and therapeutic agents which can target bone tumor and deliver drugs and genes selectively to the targeted cells. ${ }^{[15,45]}$ Targeted delivery and controlled release of chemotherapeutic agents by nanocarriers prevent rapid clearance of the drug, prolong blood circulation time, enhance intra-tumoral accumulation, thereby, improving therapeutic efficacy and minimizing side effects. ${ }^{[45]}$

A cisplatin-crosslinked, doxorubicin-loaded hyaluronic acid nanogel was reported for the effective treatment of osteosarcoma. ${ }^{[4]}$ This codelivery system exhibited a prolonged circulation time owing to the enhanced stability of the nanogel, compared to free drugs. Synergistic apoptosis-inducing effects of doxorubicin and cisplatin were observed following valid tumor accumulation, indicating its great potential for the chemotherapy of osteosarcoma. ${ }^{[46]} \mathrm{In}$ another study by Au et al., ${ }^{[47]} \mathrm{pH}$-responsive nanoscale metal-organic frameworks were developed for the delivery of calcium zoledronate for the treatment of cancer bone metastasis. Folate, a targeting ligand, was incorporated to facilitate the tumor uptake of nanoparticles. ${ }^{[15]}$ The authors demonstrated the increase in direct antitumor activity of zoledronate by 80 to $85 \%$ in vivo, compared to free drug, which could be explained by the improved tumor uptake and the prolonged drug release kinetics. ${ }^{[47]}$ Similarly, an intelligent nano drug delivery system composed of functional graphene oxide conjugated with folic acid, polyethylene glycol, and photosensitizer indocyanine green was developed for the delivery of MutT homolog 1 inhibitor and doxorubicin. This nano drug delivery system responding to low $\mathrm{pH}$ environment within a tumor and having photothermal and photodynamic transformation abilities showed combined chemo-photodynamic effects to inhibit osteosarcoma. ${ }^{[47]}$

\section{Osteoporosis}

Osteoporosis is a degenerative and progressive bone disease, affecting hundreds of millions of aged individuals worldwide, with a consequent increase in the bone fragility and susceptibility to fracture. ${ }^{[48-50]}$ Systemically administered anti-osteoporotic drugs lead to side effects, due to off-target tissue effects. Therefore, novel drugs with higher therapeutic efficacy, less adverse side effects, and more convenient administration routes are needed. On the other hand, nanostructured drug delivery systems, which are able to allow specific drug release kinetics, increase local drug concentration, reduce side effects and enhance bone regeneration, represent innovative and alternative treatment methods for osteoporosis.

A controlled drug release system consisting of simvastatin-loaded poly( $\mathrm{N}$-isopropylacrylamide) brush-modified mesoporous hydroxyapatite nanoparticles was developed for the repair of osteoporotic local defects. ${ }^{[51]}$ This system provided a sustained release of simvastatin to inhibit osteoporosis along with mesoporous hydroxyapatite nanoparticle promoting the osteogenesis as a novel strategy. Ryu et al. ${ }^{[52]}$ designed alendronateconjugated nanodiamonds and investigated for potential treatment of osteoporosis to obtain a synergistic effect as a bone-targeted delivery system. Specific accumulation to the bone tissue, high affinity to hydroxyapatite, positive synergistic effect for alkaline phosphatase activity, and in vivo bone targeting ability demonstrated the potential of alendronate-conjugated nanodiamonds for osteoporosis treatment. In another study by Nagai et al., ${ }^{[33]}$ transdermal formulations containing raloxifene solid nanoparticles were designed to improve the low bioavailability of raloxifene by using a permeation enhancer and evaluated them for osteoporosis treatment in an ovariectomized rat model. In this study, a high rate of skin penetration of raloxifene through the transdermal route and high therapeutic effects on osteoporosis were observed.

\section{Orthopedic infections}

Nanotechnology has demonstrated a great potential for the treatment and prevention of bone infections with the development of antibacterial nanomaterials to improve antibiotic efficacy and to overcome bacterial resistance in selected cases. Nanotechnology-based antibiotic delivery systems offer many advantages over free antibiotics, including controlled and sustained drug release kinetics, ease of surface modification for bacteria or bone targeting, high local bioactivity, low systemic side effects, ability to deliver multiple antibiotics and, enhanced drug solubility and stability. ${ }^{[54,55]}$

Some nanostructures such as metallic nanoparticles have intrinsic antibacterial activities. Qadri et al. ${ }^{[56]}$ utilized antibacterial activity of silvercopper-boron composite nanoparticles to eradicate Staphylococcus aureus bone infection in a mouse osteomyelitis model and demonstrated a potential inorganic route as an antibiotic free and effective alternative approach which could be used for the treatment of osteomyelitis. ${ }^{[56]}$

Mesoporous silica nanoparticles decorated with concanavalin A and loaded with levoxacin were 
designed as a new class of targeted delivery device. ${ }^{[57]}$ Covalent grafting of concanavalin A to nanoparticles provided an effective penetration in Gram-negative bacteria biofilm, leading to an increased antibacterial efficacy of levofloxacin. Synergistic combination of biofilm internalization and antibacterial agent resulted in a remarkable antibacterial effect against bacterial biofilm..$^{[57]}$

The combination of antibacterial nanomaterials with implants provides the possibility of osseointegration and elimination of infection simultaneously. ${ }^{[58]}$ Similarly, antibacterial nanomaterials can be also conjugated with bioactive matrix materials to form dual functional composites which are able to both eradicate infection and provide a scaffold for enhanced osteogenesis. ${ }^{[59,60]}$ Combination of scaffolds with nano-based drug delivery systems stands out as a successful strategy both to improve tissue regeneration and eradicate infection.

The bone cements which have been currently used have several limitations including antibacterial performance and elution of antibiotics from polymethylmethacrylate matrix. ${ }^{[6]]}$ A variety of nanotechnology-based antibiotic delivery systems have been explored to improve the drug release profile from polymethylmethacrylate bone cement including polymeric nanoparticles, carbon nanotubes, liposomes, clay nanotubes, and hydroxyapatite nanorods. ${ }^{[62-64]}$

\section{Bone regeneration}

Despite the advances in pharmacological and surgical interventions over the last years, bone regeneration and reconstruction still represent a major concern in the field of orthopedic medicine. Bone tissue engineering is considered a promising approach for bone tissue repair and regeneration using a variety of bioactive materials. ${ }^{[65]}$ Nanotechnologies appear to have an important and promising role in bone tissue engineering. The use of nanostructured scaffolds provides an appropriate environment for cell proliferation, differentiation, adhesion, and bone formation by mimicking the biological micro- and nano-environments, as well as controlled delivery of drugs and growth factors in the lesion site. Nanoparticles can be also combined with scaffolds to further facilitate the bone regeneration.

Many studies in the literature reports the use of three-dimensional nanostructured scaffolds with drug delivery capabilities for bone tissue regeneration. ${ }^{[66-70]}$ A mesoporous silicate nanoparticle incorporated-nanofibrous gelatin scaffold was designed for the dual delivery of bone morphogenic protein 2 and deferoxamine serving as a biomimetic osteogenic environment. ${ }^{[7]}$ The results showed that the designed scaffold had the ability to control the dual drug delivery of bone morphogenic protein 2 and deferoxamine at distinct release rates, while maintaining their osteogenic and angiogenic abilities, respectively.

A hybrid nanoparticle/hydrogel small interfering ribonucleic acid (siRNA) delivery system was developed for bone fracture healing as an injectable formulation. ${ }^{[72]}$ The results revealed that the formulated system was able to increase bone formation and accelerate healing significantly, which make them potential materials for fracture healing.

Carbon nanotubes are employed in tissue engineering owing to their mechanical, structural and biological properties and also used as delivery vehicles for drugs and genes. ${ }^{[73]}$ Sharmeen et al. ${ }^{[74]}$ reported a controlled drug-releasing matrix using multi-walled carbon nanotubes. The multi-walled carbon nanotube incorporated gelatin-chitosan nanocomposite films were designed and loaded with ciprofloxacin, a common antibiotic. Improved drug release capacity, as well as mechanical and thermal properties were reported by the incorporation of carbon nanotubes, compared to non-incorporated nanocomposite films.

\section{NANOTOXICOLOGY}

On the other hand, the rapid development of nanotechnology research and production of wide range of new nanomaterials for diverse applications have led to concerns regarding their potential risks on human health. Although a significant amount of research has been carried out to assess the safety of nanomaterials, there is still a need for further studies to reach the knowledge about the interaction of nanomaterials with living organisms, as well as cellular responses and long-term clinical safety. Extensive toxicological studies, in vivo investigations, and clinical trials are required to accelerate the translation of nanotechnology-based drug delivery systems into clinical practice.

In conclusion, nanotechnology-based drug delivery systems have the potential to significantly improve the treatment of various bone-related diseases. Recently, there is a number of outstanding nano-based drug delivery system applications in the treatment of bone related-diseases. Despite the considerable progress in the field of nanobased drug delivery systems for use in orthopedics, 
further extensive studies and clinical trials should be performed to demonstrate their efficacy and to evaluate their long-term safety profiles before the results are translated to the clinical practice. A close collaboration among researchers and clinicians is also essential for the achievement of an effective clinical translation.

\section{Declaration of conflicting interests}

The author declared no conflicts of interest with respect to the authorship and/or publication of this article.

\section{Funding}

The author received no financial support for the research and/or authorship of this article.

\section{REFERENCES}

1. Feynman RP. There's Plenty of Room at the Bottom. Engineering and Science 1960;23:22-36.

2. Güven E. Lipid-based nanoparticles in the treatment of erectile dysfunction. Int J Impot Res 2020;32:578-86.

3. Cacciatore FA, Brandelli A, Malheiros PDS. Combining natural antimicrobials and nanotechnology for disinfecting food surfaces and control microbial biofilm formation. Crit Rev Food Sci Nutr 2020:1-12.

4. Li C, Yan B. Opportunities and challenges of phytonanotechnology. Environmental Science: Nano 2020;7:2863-74.

5. Al Mamun MA, Yuce MR. Recent Progress in Nanomaterial Enabled Chemical Sensors for Wearable Environmental Monitoring Applications. Adv Funct Mater. 2020. ARTN 2005703.

6. Alp E, Damkaci F, Guven E, Tenniswood M. Starch nanoparticles for delivery of the histone deacetylase inhibitor CG-1521 in breast cancer treatment. Int J Nanomedicine 2019;14:1335-46.

7. Zhang H, Fan T, Chen W, Li Y, Wang B. Recent advances of two-dimensional materials in smart drug delivery nanosystems. Bioact Mater 2020;5:1071-86.

8. Deng $\mathrm{Y}$, Zhang $\mathrm{X}$, Shen $\mathrm{H}, \mathrm{He} \mathrm{Q}, \mathrm{Wu} \mathrm{Z}$, Liao W, et al. Application of the nano-drug delivery system in treatment of cardiovascular diseases. Front Bioeng Biotechnol 2020;7:489.

9. Oroojalian F, Charbgoo F, Hashemi M, Amani A, YazdianRobati R, Mokhtarzadeh A, et al. Recent advances in nanotechnology-based drug delivery systems for the kidney. J Control Release 2020;321:442-62.

10. Masoudi Asil S, Ahlawat J, Guillama Barroso G, Narayan M. Nanomaterial based drug delivery systems for the treatment of neurodegenerative diseases. Biomater Sci 2020;8:4109-28.

11. Shi J, Votruba AR, Farokhzad OC, Langer R. Nanotechnology in drug delivery and tissue engineering: from discovery to applications. Nano Lett 2010;10:3223-30.

12. Kavaz D, Odabas S, Guven E, Demirbilek M, Denkbas EB. Bleomycin Loaded Magnetic Chitosan Nanoparticles as Multifunctional Nanocarriers. Journal of Bioactive and Compatible Polymers 2010;25:305-18.

13. Patra JK, Das G, Fraceto LF, Campos EVR, Rodriguez-Torres MDP, Acosta-Torres LS, et al. Nano based drug delivery systems: recent developments and future prospects. J Nanobiotechnology 2018;16:71.

14. Kumar S, Nehra M, Kedia D, Dilbaghi N, Tankeshwar K, KimKH.Nanotechnology-basedbiomaterialsfororthopaedic applications: Recent advances and future prospects. Mater Sci Eng C Mater Biol Appl 2020;106:110154.

15. Alp E, Çirak T, Demirbilek M, Türk M, Güven E. Targeted delivery of etoposide to osteosarcoma cells using poly(3-hydroxybutyrate-co-3-hydroxyvalerate) (PHBV) nanoparticles. Turk J Biol 2017;41:719-33.

16. Sun H, Lv L, Bai Y, Yang H, Zhou H, Li C, et al. Nanotechnology-enabled materials for hemostatic and anti-infection treatments in orthopedic surgery. Int J Nanomedicine 2018;13:8325-38.

17. Parizek M, Douglas TE, Novotna K, Kromka A, Brady MA, Renzing A, et al. Nanofibrous poly(lactide-coglycolide) membranes loaded with diamond nanoparticles as promising substrates for bone tissue engineering. Int J Nanomedicine 2012;7:1931-51.

18. Yun YH, Eteshola E, Bhattacharya A, Dong Z, Shim JS, Conforti L, et al. Tiny medicine: nanomaterial-based biosensors. Sensors (Basel) 2009;9:9275-99.

19. Raina DB, Liu Y, Jacobson OLP, Tanner KE, Tagil M, Lidgren L. Bone mineral as a drug-seeking moiety and a waste dump a Review. Bone Joint Res 2020;9:709-18.

20. Chen X, Zhu Q, Xu X, Shen S, Zhang Y, Mo R. Sequentially site-specific delivery of apoptotic protein and tumorsuppressor gene for combination cancer therapy. Small 2019;15:e1902998.

21. Yang Y, Sun B, Zuo S, Li X, Zhou S, Li L, et al. Trisulfide bondmediated doxorubicin dimeric prodrug nanoassemblies with high drug loading, high self-assembly stability, and high tumor selectivity. Sci Adv 2020;6:eabc1725.

22. Zhou X, He X, Shi K, Yuan L, Yang Y, Liu Q, et al. Injectable thermosensitive hydrogel containing erlotinib-loaded hollow mesoporous silica nanoparticles as a localized drug delivery system for NSCLC Therapy. Adv Sci (Weinh) 2020;7:2001442.

23. Lu C, Han HD, Mangala LS, Ali-Fehmi R, Newton CS, Ozbun L, et al. Regulation of tumor angiogenesis by EZH2. Cancer Cell 2010;18:185-97.

24. Soiberman U, Kambhampati SP, Wu T, Mishra MK, Oh Y, Sharma R, et al. Subconjunctival injectable dendrimerdexamethasone gel for the treatment of corneal inflammation. Biomaterials 2017;125:38-53.

25. Bhirde AA, Patel S, Sousa AA, Patel V, Molinolo AA, Ji $Y$, et al. Distribution and clearance of PEG-single-walled carbon nanotube cancer drug delivery vehicles in mice. Nanomedicine (Lond) 2010;5:1535-46.

26. Aizik G, Waiskopf N, Agbaria M, Ben-David-Naim M, LeviKalisman Y, Shahar A, et al. Liposomes of quantum dots configured for passive and active delivery to tumor tissue. Nano Lett 2019;19:5844-52.

27. Dave V, Sharma R, Gupta C, Sur S. Folic acid modified gold nanoparticle for targeted delivery of Sorafenib tosylate towards the treatment of diabetic retinopathy. Colloids Surf B Biointerfaces 2020;194:111151.

28. Jin T, Wu D, Liu XM, Xu JT, Ma BJ, Ji Y, et al. Intraarticular delivery of celastrol by hollow mesoporous silica nanoparticles for $\mathrm{pH}$-sensitive anti-inflammatory therapy against knee osteoarthritis. J Nanobiotechnology 2020;18:94.

29. Mao Y, Zhao Y, Guan J, Guan J, Ye T, Chen Y, et al. Electrospun fibers: an innovative delivery method for 
the treatment of bone diseases. Expert Opin Drug Deliv 2020;17:993-1005.

30. Murthy A, Ravi PR, Kathuria H, Malekar S. Oral Bioavailability Enhancement of Raloxifene with Nanostructured Lipid Carriers. Nanomaterials (Basel) 2020;10:1085.

31. Li K, Li D, Zhao L, Chang Y, Zhang Y, Cui Y, et al. Calciummineralized polypeptide nanoparticle for intracellular drug delivery in osteosarcoma chemotherapy. Bioact Mater 2020;5:721-31.

32. Hassani Besheli N, Mottaghitalab F, Eslami M, Gholami M, Kundu SC, Kaplan DL, et al. Sustainable release of vancomycin from silk fibroin nanoparticles for treating severe bone infection in rat tibia osteomyelitis model. ACS Appl Mater Interfaces 2017;9:5128-38.

33. Evans CH, Kraus VB, Setton LA. Progress in intra-articular therapy. Nat Rev Rheumatol 2014;10:11-22.

34. Rahmani Del Bakhshayesh A, Akbarzadeh A, Alihemmati A, Tayefi Nasrabadi H, Montaseri A, Davaran S, et al. Preparation and characterization of novel antiinflammatory biological agents based on piroxicam-loaded poly- $\varepsilon$-caprolactone nano-particles for sustained NSAID delivery. Drug Deliv 2020;27:269-82.

35. Sacchetti C, Liu-Bryan R, Magrini A, Rosato N, Bottini N, Bottini M. Polyethylene-glycol-modified singlewalled carbon nanotubes for intra-articular delivery to chondrocytes. ACS Nano 2014;8:12280-91.

36. McMasters J, Poh S, Lin JB, Panitch A. Delivery of anti-inflammatory peptides from hollow PEGylated poly(NIPAM) nanoparticles reduces inflammation in an ex vivo osteoarthritis model. J Control Release 2017;258:161-70.

37. Yan Y, Sun T, Zhang H, Ji X, Sun Y, Zhao X, et al. Euryale ferox seed-inspired superlubricated nanoparticles for treatment of osteoarthritis. Advanced Functional Materials 2019;29:1807559.

38. Lin JB, Poh S, Panitch A. Controlled release of antiinflammatory peptides from reducible thermosensitive nanoparticles suppresses cartilage inflammation. Nanomedicine 2016;12:2095-100.

39. Maudens P, Seemayer CA, Thauvin C, Gabay C, Jordan O, Allémann E. Nanocrystal-polymer particles: Extended delivery carriers for osteoarthritis treatment. Small 2018;14.

40. Johnson K, Zhu S, Tremblay MS, Payette JN, Wang J, Bouchez LC, et al. A stem cell-based approach to cartilage repair. Science 2012;336:717-21.

41. Kang ML, Kim JE, Im GI. Thermoresponsive nanospheres with independent dual drug release profiles for the treatment of osteoarthritis. Acta Biomater 2016;39:65-78.

42. He T, Zhang C, Vedadghavami A, Mehta S, Clark HA, Porter RM, et al. Multi-arm Avidin nano-construct for intra-cartilage delivery of small molecule drugs. J Control Release 2020;318:109-23.

43. Bajpayee AG, Scheu M, Grodzinsky AJ, Porter RM. A rabbit model demonstrates the influence of cartilage thickness on intra-articular drug delivery and retention within cartilage. J Orthop Res 2015;33:660-7.

44. Steckiewicz KP, Inkielewicz-Stepniak I. Modified nanoparticles as potential agents in bone diseases: Cancer and implant-related complications. Nanomaterials (Basel) 2020;10:658.

45. Kılıçay E, Demirbilek M, Türk M, Güven E, Hazer B, Denkbas EB. Preparation and characterization of poly(3hydroxybutyrate-co-3-hydroxyhexanoate) (PHBHHX) based nanoparticles for targeted cancer therapy. Eur J Pharm Sci 2011;44:310-20.

46. Zhang Y, Wang F, Li M, Yu Z, Qi R, Ding J, et al. Erratum: Self-stabilized hyaluronate nanogel for intracellular codelivery of doxorubicin and cisplatin to osteosarcoma. Adv Sci (Weinh) 2018;5:1800811.

47. Au KM, Satterlee A, Min Y, Tian X, Kim YS, Caster JM, et al. Folate-targeted $\mathrm{pH}$-responsive calcium zoledronate nanoscale metal-organic frameworks: Turning a bone antiresorptive agent into an anticancer therapeutic. Biomaterials 2016;82:178-93.

48. Bozkurt HH, Tokgöz MA, Yapar A, Atik OŞ. What is the importance of canal-to-diaphysis ratio on osteoporosis-related hip fractures? Eklem Hastalik Cerrahisi 2019;30:296-300.

49. Wei D, Jung J, Yang H, Stout DA, Yang L. Nanotechnology treatment options for osteoporosis and its corresponding consequences. Curr Osteoporos Rep 2016;14:239-47.

50. Wang J, Tao S, Jin X, Song Y, Zhou W, Lou H, et al. Calcium supplement by tetracycline guided amorphous calcium carbonate potentiates osteoblast promotion for synergetic osteoporosis therapy. Theranostics 2020;10:8591-605.

51. Wu T, Sun J, Tan L, Yan Q, Li L, Chen L, et al. Enhanced osteogenesis and therapy of osteoporosis using simvastatin loaded hybrid system. Bioact Mater 2020;5:348-57.

52. Ryu TK, Kang RH, Jeong KY, Jun DR, Koh JM, Kim $\mathrm{D}$, et al. Bone-targeted delivery of nanodiamond-based drug carriers conjugated with alendronate for potential osteoporosis treatment. J Control Release 2016;232:152-60.

53. Nagai N, Ogata F, Otake H, Nakazawa Y, Kawasaki N. Design of a transdermal formulation containing raloxifene nanoparticles for osteoporosis treatment. Int J Nanomedicine 2018;13:5215-29.

54. Guo P, Xue HY, Wong HL. Therapeutic nanotechnology for bone infection treatment - state of the art. Curr Drug Deliv 2018;15:941-52.

55. Qayoom I, Verma R, Murugan PA, Raina DB, Teotia AK, Matheshwaran S, et al. A biphasic nanohydroxyapatite/ calcium sulphate carrier containing Rifampicin and Isoniazid for local delivery gives sustained and effective antibiotic release and prevents biofilm formation. Sci Rep 2020;10:14128.

56. Qadri S, Haik Y, Mensah-Brown E, Bashir G, FernandezCabezudo MJ, Al-Ramadi BK. Metallic nanoparticles to eradicate bacterial bone infection. Nanomedicine 2017;13:2241-50.

57. Martínez-Carmona M, Izquierdo-Barba I, Colilla M, Vallet-Regí M. Concanavalin A-targeted mesoporous silica nanoparticles for infection treatment. Acta Biomater 2019;96:547-56.

58. Song W, Seta J, Chen L, Bergum C, Zhou Z, Kanneganti $\mathrm{P}$, et al. Doxycycline-loaded coaxial nanofiber coating of titanium implants enhances osseointegration and inhibits Staphylococcus aureus infection. Biomed Mater 2017;12:045008.

59. Wang $Y$, Jiang $Y$, Zhang $Y$, Wen S, Wang $Y$, Zhang $H$. Dual functional electrospun core-shell nanofibers for antiinfective guided bone regeneration membranes. Mater Sci Eng C Mater Biol Appl 2019;98:134-9.

60. Stravinskas M, Tarasevicius S, Laukaitis S, Nilsson M, Raina DB, Lidgren L. A ceramic bone substitute containing gentamicin gives good outcome in trochanteric hip fractures treated with dynamic hip screw and in revision 
of total hip arthroplasty: a case series. BMC Musculoskelet Disord 2018;19:438.

61. Atıcı T, Şahin N, Çavun S, Özakin C, Kaleli T. Antibiotic release and antibacterial efficacy in cement spacers and cement beads impregnated with different techniques: In vitro study. Eklem Hastalik Cerrahisi 2018;29:71-8.

62. Al Thaher Y, Perni S, Prokopovich P. Nano-carrier based drug delivery systems for sustained antimicrobial agent release from orthopaedic cementous material. Adv Colloid Interface Sci 2017;249:234-47.

63. Çağlar Ö, Tokgözoğlu M, Akgün RC, Atilla B. Lowdose vancomycin-loaded cement spacer for two-stage revision of infected total hip arthroplasty. Jt Dis Relat Surg 2020;31:449-55.

64. Aşık MD, Kaplan M, Yalınay M, Güven EÖ, Bozkurt M. Development of a sequential antibiotic releasing system for two-stage total joint replacement surgery. J Biomed Nanotechnol 2019;15:2193-201.

65. Raina DB, Matuszewski LM, Vater C, Bolte J, Isaksson H, Lidgren $\mathrm{L}$, et al. A facile one-stage treatment of critical bone defects using a calcium sulfate/hydroxyapatite biomaterial providing spatiotemporal delivery of bone morphogenic protein-2 and zoledronic acid. Sci Adv 2020;6:eabc1779.

66. J Hill M, Qi B, Bayaniahangar R, Araban V, Bakhtiary $Z$, Doschak MR, et al. Nanomaterials for bone tissue regeneration: updates and future perspectives. Nanomedicine (Lond) 2019;14:2987-3006.

67. Cheng X, Cheng G, Xing X, Yin C, Cheng Y, Zhou X, et al. Controlled release of adenosine from core-shell nanofibers to promote bone regeneration through STAT3 signaling pathway. J Control Release 2020;319:234-45.

68. Cheng G, Yin C, Tu H, Jiang S, Wang Q, Zhou X, et al. Controlled co-delivery of growth factors through layer-bylayer assembly of core-shell nanofibers for improving bone regeneration. ACS Nano 2019;13:6372-82.

69. Limongi T, Susa F, Allione M, di Fabrizio E. Drug delivery applications of three-dimensional printed (3DP) mesoporous scaffolds. Pharmaceutics 2020;12:851.

70. Bhattarai DP, Kim MH, Park H, Park WH, Kim BS, Kim CS. Coaxially fabricated polylactic acid electrospun nanofibrous scaffold for sequential release of tauroursodeoxycholic acid and bone morphogenic protein 2 to stimulate angiogenesis and bone regeneration. Chemical Engineering Journal 2020;389:123470.

71. Yao Q, Liu Y, Selvaratnam B, Koodali RT, Sun H. Mesoporous silicate nanoparticles/3D nanofibrous scaffold-mediated dual-drug delivery for bone tissue engineering. J Control Release 2018;279:69-78.

72. Wang Y, Malcolm DW, Benoit DSW. Controlled and sustained delivery of siRNA/NPs from hydrogels expedites bone fracture healing. Biomaterials 2017;139:127-38.

73. Yang L, Zhang L, Webster TJ. Carbon nanostructures for orthopedic medical applications. Nanomedicine (Lond) 2011;6:1231-44.

74. Sharmeen S, Rahman AFMM, Lubna MM, Salem KS, Islam $\mathrm{R}$, Khan MA. Polyethylene glycol functionalized carbon nanotubes/gelatin-chitosan nanocomposite: An approach for significant drug release. Bioact Mater 2018;3:236-44. 